\title{
Nanoparticles produced by nanosecond pulse laser ablation of a metallic glass in water
}

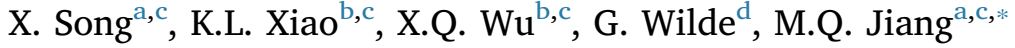 \\ ${ }^{a}$ State Key Laboratory of Nonlinear Mechanics, Institute of Mechanics, Chinese Academy of Sciences, Beijing 100190, PR China \\ ${ }^{\mathrm{b}}$ Key Laboratory of Mechanics in Fluid Solid Coupling Systems, Institute of Mechanics, Chinese Academy of Sciences, Beijing 100190, PR China \\ ${ }^{\mathrm{c}}$ School of Engineering Science, University of Chinese Academy of Sciences, Beijing 100049, PR China \\ ${ }^{\mathrm{d}}$ Institute of Materials Physics, Westfälische Wilhelms-Universität Münster, Münster 48149, Germany
}

\section{A R T I C L E I N F O}

\section{Keywords:}

Metallic glasses

Pulse laser ablation

Nanoparticles

Cavitation bubble

Core-shell structures

\begin{abstract}
A B S T R A C T
In this paper, we perform a single nanosecond pulse laser ablation of a Zr-based metallic glass (Vitreloy 1) target in water. The violent ejection of high-temperature ablation matter is observed from the target by means of explosive boiling, which is accompanied by a formation of cavitation bubble. These ablation products entered the water include a rich variety of nanoparticles that can be classified into three different types: full amorphous, amorphous-crystalline composite, and polycrystalline. The amorphous nanoparticles have relatively smaller sizes and a uniform elemental distribution. The latter two types of nanoparticles exhibit a unique core-shell feature with an obvious compositional segregation. It is proposed that the diversity of the nanoparticles closely depends on the different pathways that they enter the water: directly before the bubble formation or via the bubble, in which the cooling rate and the glass-forming ability of ablation products are two competing factors.
\end{abstract}

\section{Introduction}

The first laser device was built in 1960 [1], and the metallic glass was first discovered in the same year [2]. For the past decades, lasers are commonly used as a processing technology of metallic glasses [3-11], which expands their applicability as advanced structural or functional materials. In recent years, the mechanism for pulse laser ablation (PLA) of metallic glasses has attracted increasing attention [12-16]. When a laser pulse is applied a solid target, complex physical and mechanical processes occur, which involves plasma formation $[17,18]$, athermal or thermal ablation $[15,16,19]$, shock wave [20,21], etc. For nanosecond PLA of metallic glasses, the thermal ablation is dominated via normal vaporization and boiling or phase explosion, depending on the applied laser energy fluence. It is experimentally shown that, compared with crystalline targets, metallic glasses are prone to undergo superheating and explosive boiling [14]. This critical phenomenon is evidenced by the violent ejection of high-temperature matter from the target and the formation of a liquid-gas spinodal ablation pattern. The similar mechanism is also active in the underwater PLA of metallic glasses, although an additional cavitation bubble can be observed on the target surface [22]. Usually, the ejected matter can be captured by the bubble and gets eventually delivered into the water after the bubble has collapse. This provides a relatively simple and feasible route to produce nanoparticles with desirable structures and properties [23-26]. For metallic glass targets, however, it is still unknown whether nanoparticles can be formed from the ejected ablation matter into water. What are their structures or compositions if the answer is affirmative? These questions have never been verified so far, which deserves further investigations to provide additional evidence for the ablation mechanism revealed previously.

In this work, we perform the nanosecond PLA of a Vitreloy 1 metallic glass target in water, during which both the ejection of ablation matter and the cavitation bubble are real-time observed in situ. For the first time, three types of nanoparticles with full amorphous, crystallineamorphous composite, or completely polycrystalline structures are found in the ablation products that eventually enter the water. The possible formation mechanism for these nanoparticles has been briefly discussed as well.

\section{Experimental}

A typical $\mathrm{Zr}_{41.2} \mathrm{Ti}_{13.8} \mathrm{Cu}_{12.5} \mathrm{Ni}_{22.5} \mathrm{Be}_{10.0}$ (Vitreloy 1) metallic glass was used as the target material, because of its good glass-forming ability (GFA) and relatively high thermal stability [27]. The surfaces of the targets $(10 \mathrm{~mm} \times 10 \mathrm{~mm} \times 2 \mathrm{~mm})$ were polished to remove oxides. The amorphous structure of the targets was confirmed by both X-ray

\footnotetext{
* Corresponding author at: State Key Laboratory of Nonlinear Mechanics, Institute of Mechanics, Chinese Academy of Sciences, Beijing 100190, PR China.

E-mail address: mqjiang@imech.ac.cn (M.Q. Jiang).
} 


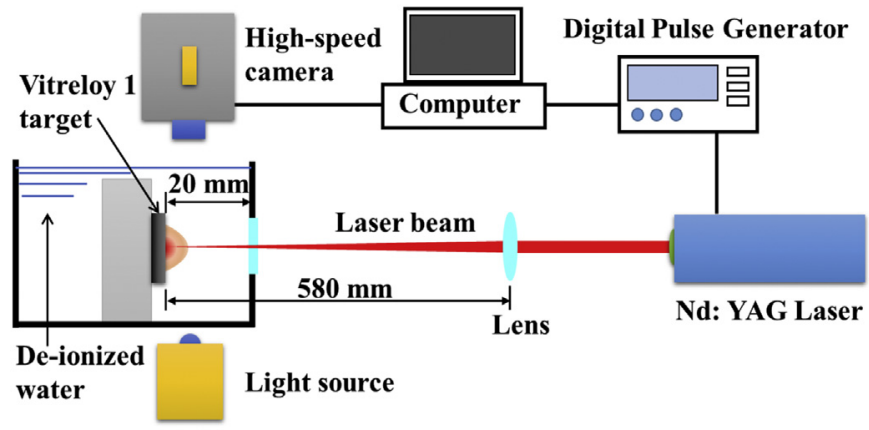

Fig. 1. Schematic of the experimental setup.

diffraction and differential scanning calorimetry. Single PLA experiments were performed by irradiation with a Q-switched Nd:YAG laser operating at a wavelength of $1064 \mathrm{~nm}$ and a maximum output energy of $2.5 \mathrm{~J}$ per shot. The temporal profile of the laser pulse is in the nearGaussian distribution with a full width of at half maximum (FWHM) of about 10 ns. A schematic of the experimental setup is shown in Fig. 1. The incident laser beam was focused by a quartz lens with a focal distance of $660 \mathrm{~mm}$ normally to the target that was placed inside a vessel with $80 \mathrm{ml}$ of filtered, de-ionized water. The distance between the lens and the target is $580 \mathrm{~mm}$, and the beam path length in water is about $20 \mathrm{~mm}$, minimizing the possibility of nonlinear absorption of the laser in water. A high speed camera (Phantom v2512) with a framing rate of 50,000 fps was used to observe the process of laser ablation. A signal generator was applied to trigger the laser beam and the highspeed camera simultaneously.

After ablation, the surface morphologies of the targets were examined by using FEI Quanta 200 scanning electron microscope (SEM) equipped with an energy dispersive X-ray spectroscopy (EDS). Structural characterizations of the ablation products were performed by a field emission high-resolution transmission electron microscope (HRTEM, JEOL 2100F) operated at $200 \mathrm{keV}$. The composition and elemental distribution of the ablation products were further mapped through EDS by displaying the integrated intensities of the signals of the individual elements as a function of the beam position when operating the TEM in scanning mode (STEM). For the TEM observations, the suspension containing the ablation products was dropped on a carboncoated copper grid using transferpette.

\section{Results and discussion}

Fig. 2 (and Supplementary Movie S1, Appendix A) shows the typical underwater-PLA process recorded by the high-speed camera, and the characteristic stages along a time-axis are illustrated at the bottom of Fig. 2. The second snapshot captures the optically active plasma plume that has already expanded into the entire viewing window. For the ns pulse width, an amount of laser energy can be absorbed by the plasma plume, i.e., the so-called "plasma shielding" effect [28]. Nevertheless,

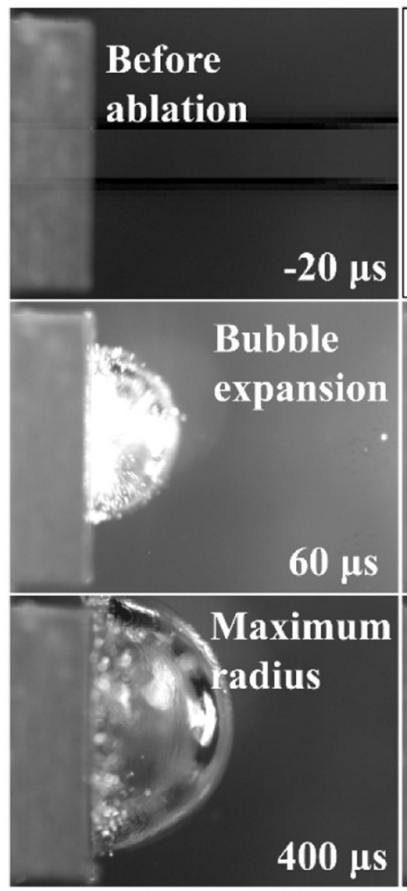

\section{Plasma plume}

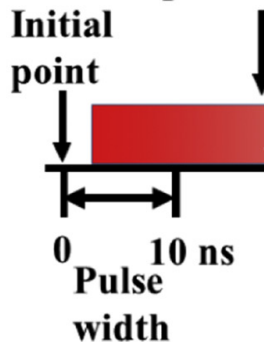

boiling
$0 \mu \mathrm{s}$

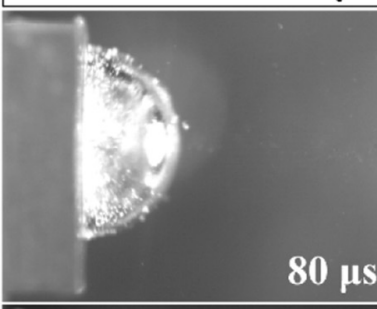

Bubble shrink

$800 \mu \mathrm{s}$
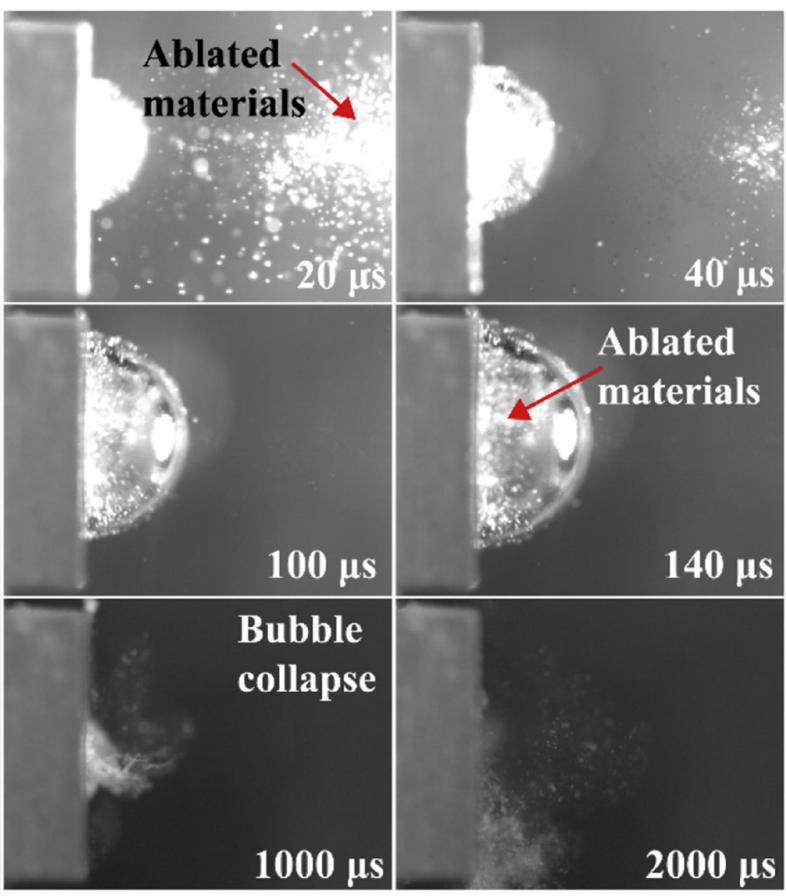
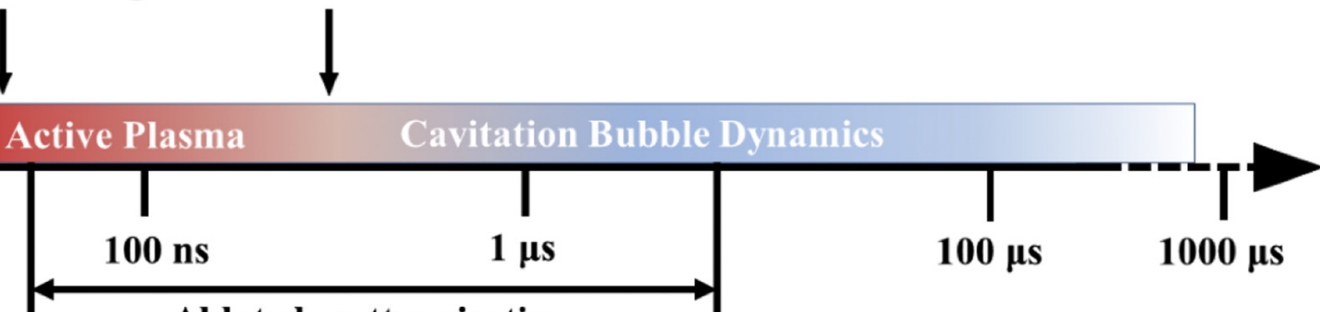

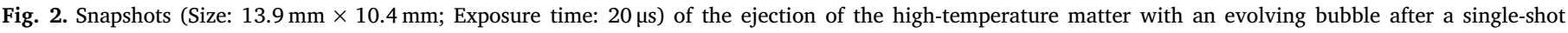
nanosecond PLA of the Vitreloy 1 target in water. The sketch at the bottom of the figure shows the main stages during the PLA. 

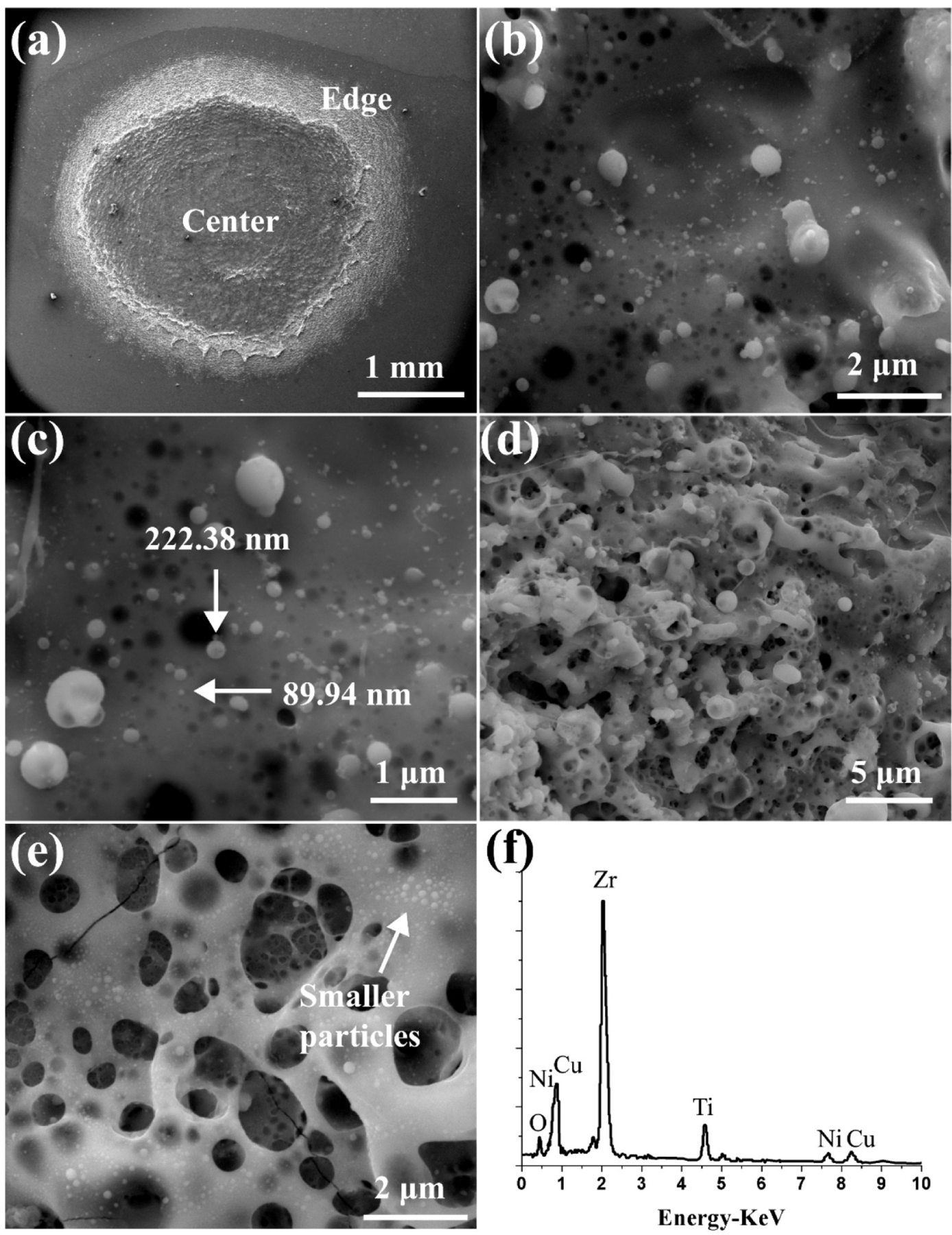

Fig. 3. Surface morphologies of the Vitreloy 1 target after underwater PLA. (a) Full view of the ablation area. Ablation voids and molten droplets observed in the central ( $b$ and $c$ ) and the edge ( $d$ and e) regions of the ablation area. (f) SEM-EDS result of molten droplets.

the lifetime of the plasma plume is still much shorter than the time $(20 \mu \mathrm{s})$ between two successional frames. As a result, we only capture one snapshot about the plasma. It is reasonable to take this time as the beginning of the snapshots $(t=0 \mu \mathrm{s})$. According to our previous study [22], the plasma pressure will increase rapidly to a peak value within about a pulse-width time. After the extinguishing of the laser pulse, the plasma cools down and its pressure and volume attain to a relatively stable state, which induces the nucleation of a cavitation bubble. Due to the mirror symmetry on the flat surface of the target [29], the dynamic evolution of the bubble can be approximately described by the Rayleigh-Plesset equation [22,30,31]. The entire bubble dynamics process lasts about a few hundred microseconds. In addition to the cavitation bubble, we observed that a large amount of small-scaled and bright high-temperature matter is vigorously ejected from the target surface into water. This phenomenon can last for several tens of microseconds, which is ascribed to the explosive boiling [14]. By absorbing energy from the laser pulse, the amorphous target is rapidly heated up to the thermodynamic critical temperature. At this critical point, the superheated liquid boils in an explosive-like manner, and a mixture composed of high-temperature vapor and droplets is ejected violently into the water.

As shown at the bottom of Fig. 2, there is a time overlap for the explosive boiling and the cavitation bubble. The explosive boiling usually occurs with a time lag on the same as the pulse width after the laser extinguished [14,32]. The nucleation of the cavitation bubble occurs usually several hundred nanoseconds after the laser extinguished 

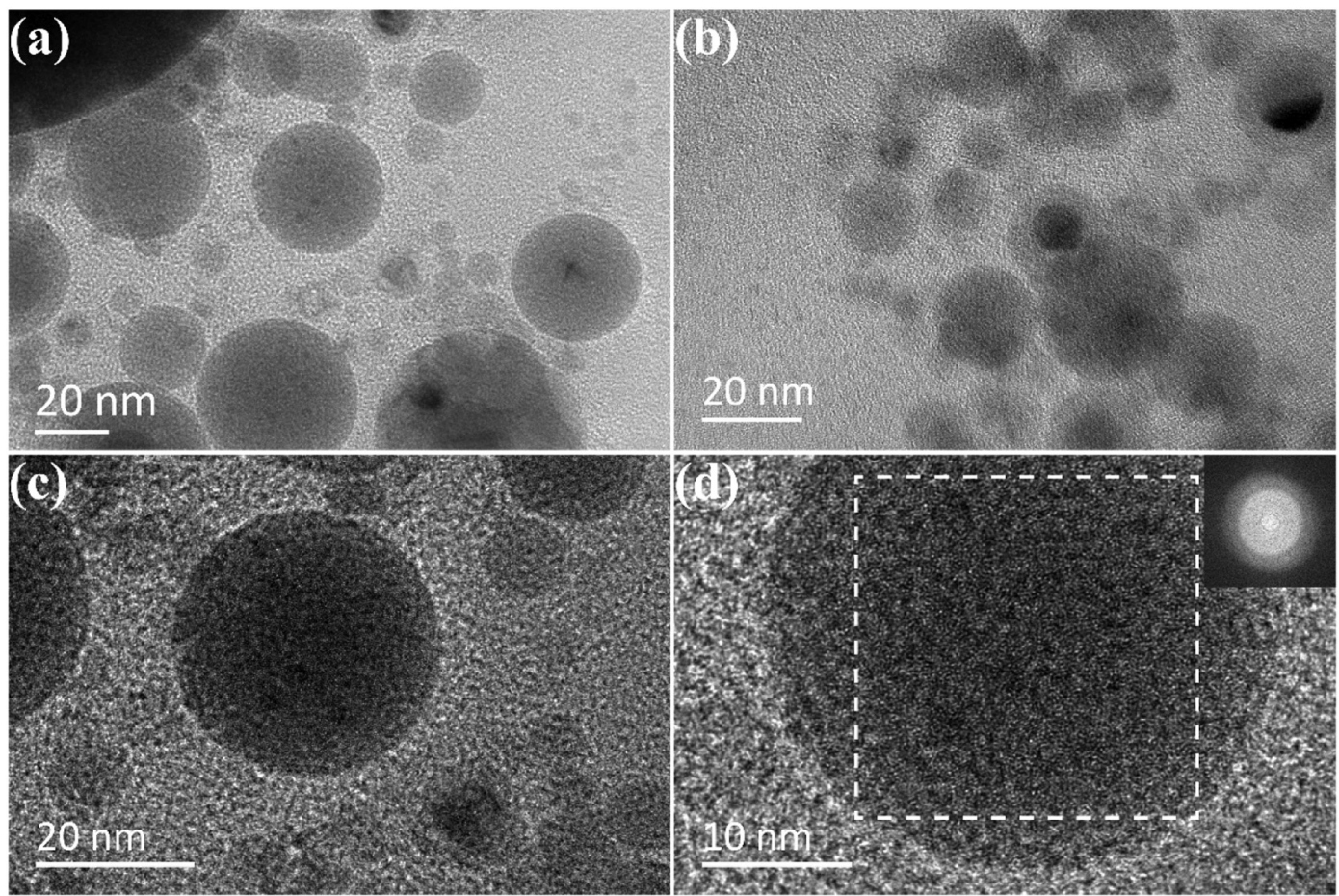

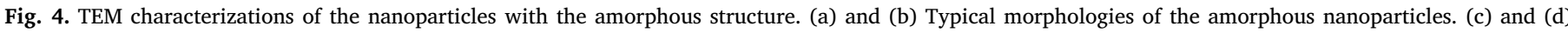
HRTEM images with the electron diffraction pattern in the inset of (d).

[22]. Therefore, the explosive boiling starts before the bubble nucleation, and covers the initial stage of the bubble dynamics. In other words, a part of the ablation products can directly enter the water before the bubble forms. This is confirmed by the snapshot $(20 \mu \mathrm{s})$ of Fig. 2, where a large amount of bright high-temperature matter is present in water beyond the bubble front. The remaining fraction of the ablation products cannot directly enter into the water, instead they will be trapped firstly by the cavitation bubble. With the expansion of the bubble, the bright ablation products that reside in the bubble cool down gradually. After reaching its maximum radius (the snapshot of $400 \mu \mathrm{s}$ ), the bubble starts to shrink. Eventually, the stored ablation products destroy the bubble and are released into the water, leading to the collapse of the bubble without rebound. It is also noticed that, during the initial expansion stage of the bubble, a few ablation products with particle-like appearance rapidly penetrate through the boundary of the bubble. Based on our observations, there are at least three different pathways for the ablation products to enter the water: before the bubble formation, during the bubble expansion, and after the bubble collapse.

Fig. 3a shows the full-view of the typical surface morphologies of the Vitreloy 1 target after the underwater PLA. By measuring the actual ablation area, the laser fluence can be estimated to be about $28 \mathrm{~J} / \mathrm{cm}^{2}$, and the corresponding laser intensity is about $2.8 \mathrm{GW} / \mathrm{cm}^{2}$. The entire ablation area can be divided into a relatively flat central region and a frilled edge region. The close-up views of the two regions are displayed in Fig. 3(b-c) and (d-e), respectively. In both regions, many ablation voids are observed, indicating the occurrence of the explosive boiling. Importantly, we observe many molten droplets on the micro-scale, and large numbers of particles with sizes ranging from several to several hundreds of nanometers. Those nanoparticles are very difficult to be observed by SEM. It is reasonable to believe that these droplets or particles belong to the ablation products that are ejected from the target surface, but deposited back on the ablation area due to the long-term confinement of the bubble. Fig. 3f gives the SEM-EDS result for these droplets or particles. The peaks of $\mathrm{Zr}, \mathrm{Ti}, \mathrm{Cu}$, and $\mathrm{Ni}$ elements are identified, but the lightest element Be cannot be detected by EDS. Compared to the initial composition of Vitreloy 1 , the element $O$ is additional, and its atomic ratio exceeds $20 \%$, which most probably stems from the confined water or its vapor. This result demonstrates that the ablation products experience an oxidation reaction due to the high-temperature-and-pressure (HTP) environment during the underwater PLA [33].

As observed in Fig. 2, the vast majority of ablation products are released into the surrounding water via three different pathways. The nanoparticles that are in the focus of this work are expected to suspend in the water. After transferred on to a carbon-coated copper grid, the nanoparticles were carefully characterized by TEM. According to their morphologies, two types of nanoparticles exist: One is structurally homogeneous, and the other displays an obvious core-shell structure. The former is of a diameter usually less than $30 \mathrm{~nm}$. But the diameter of the latter can reach about $100 \mathrm{~nm}$. Moreover, all nanoparticles are structurally stable when they are exposed to the TEM electron beam, similar to an amorphous nanofilm of Vitreloy 1 [34] or other low-dimension structures of metallic glasses [35-40].

Fig. $4 \mathrm{a}$ and $\mathrm{b}$ shows the typical nanoparticles with a homogeneous structure. The HRTEM images of these nanoparticles are presented in Fig. $4 \mathrm{c}$ and d. Obviously, these nanoparticles display a mazelike pattern without any crystalline fringes, indicating their amorphous structures. The inset of Fig. 4d shows the electron diffraction pattern of the nanoparticle by fast Fourier transformation (FFT) of the dashed square region. A halo ring is obtained, which further confirms that these homogeneous nanoparticles are full amorphous.

Fig. 5a and b shows typical nanoparticles with core-shell structures. HRTEM observations indicate that there are three kinds of core-shell nanoparticles. The most frequently observed structures consist of amorphous shells and polycrystalline cores, as presented in Fig. $5 \mathrm{c}$ and d. The results clearly show that the electron diffraction pattern of shells is a full halo ring without any detectable diffraction spots. The cores of those nanoparticles show a polycrystalline structure with different crystallographic orientations and interplanar spacings. The second kind of core-shell nanoparticles (Fig. 5e) has single-crystal cores, but the shells still remain amorphous. This is confirmed by Fig. 5f, where the single-crystal diffraction spots are identified as a FCC structure. Fig. $5 \mathrm{~g}$ 

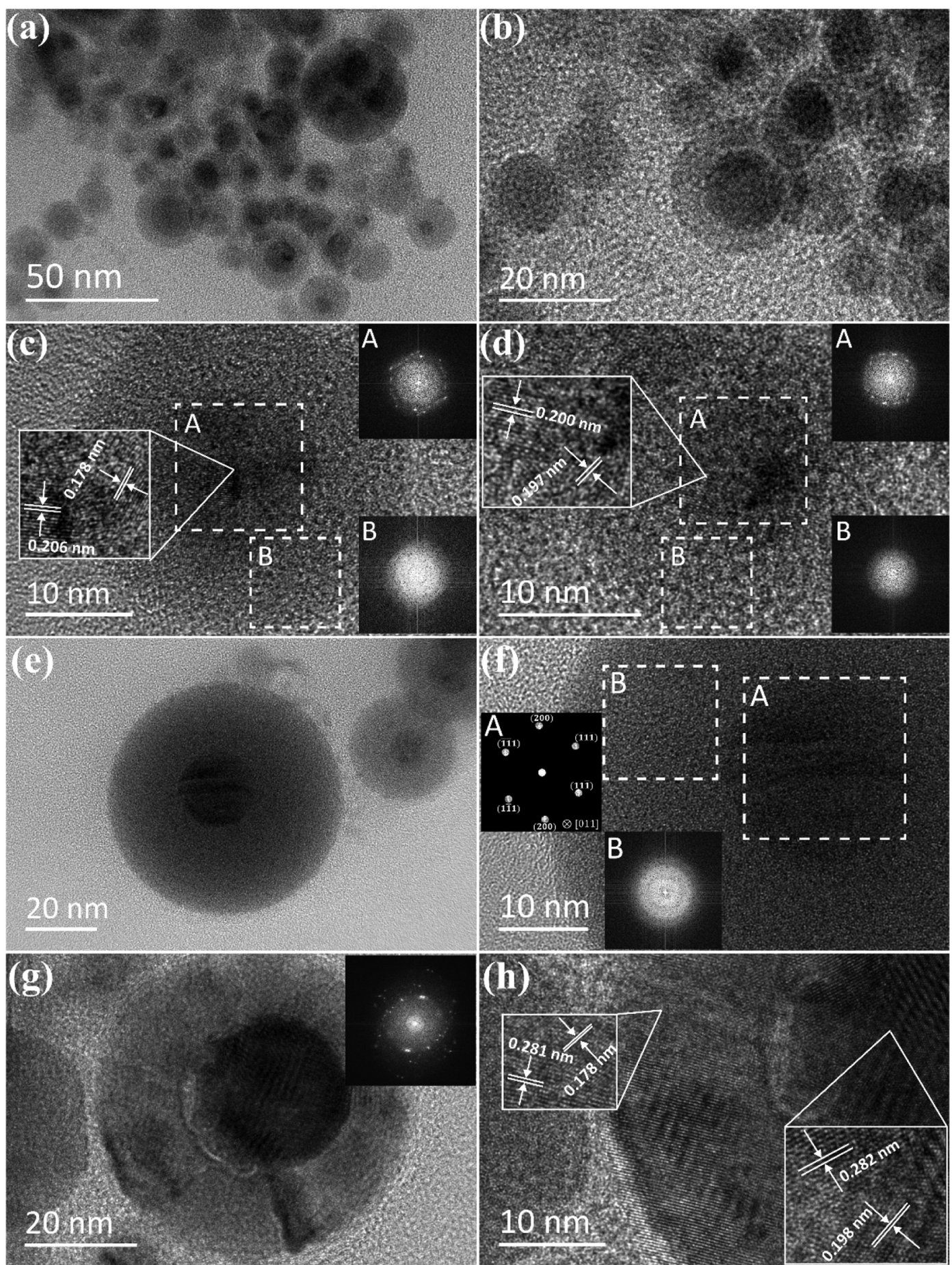

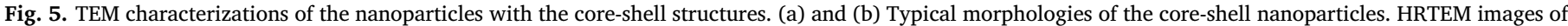

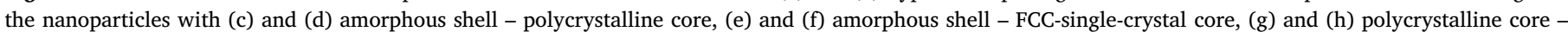
shell. Insets show the electron diffraction patterns of the corresponding dashed square regions. The interplanar spacings are indicated by white parallel lines.

presents the last kind of nanoparticles with polycrystalline core-shell structures, and the entire nanoparticle displays complex distributions of diffraction spots, indicating the polycrystalline nature of the entire nanoparticle, as shown in the inset. Multiple-oriented crystalline lattices with different interplanar spacings are distinguished in both core and shell, as marked in the insets of Fig. 5h. It is noteworthy that the latter two kinds of nanoparticles are rarely observed.

Fig. 6 shows the size distribution of both amorphous and core-shell nanoparticles. We find that the number of amorphous nanoparticles more than triples that of core-shell, but the average diameter of the latter about doubles that of the former. Most of amorphous nanoparticles are smaller than $20 \mathrm{~nm}$ in diameter, and the diameters of coreshell nanoparticles are mainly concentrated in the range of $15-45 \mathrm{~nm}$. These amorphous nanoparticles with sizes of a few nanometers are expected to be building blocks for the synthesis of nanoglasses, a new type of nanostructured solids beyond the limits of nanocrystalline materials $[41,42]$.

Fig. 7a shows that amorphous nanoparticles have a uniform distribution of the chemical compositions. There is no obvious elemental segregation for $\mathrm{Zr}, \mathrm{Ti}, \mathrm{Cu}, \mathrm{Ni}$, and $\mathrm{O}$. Again, the Be element cannot be 

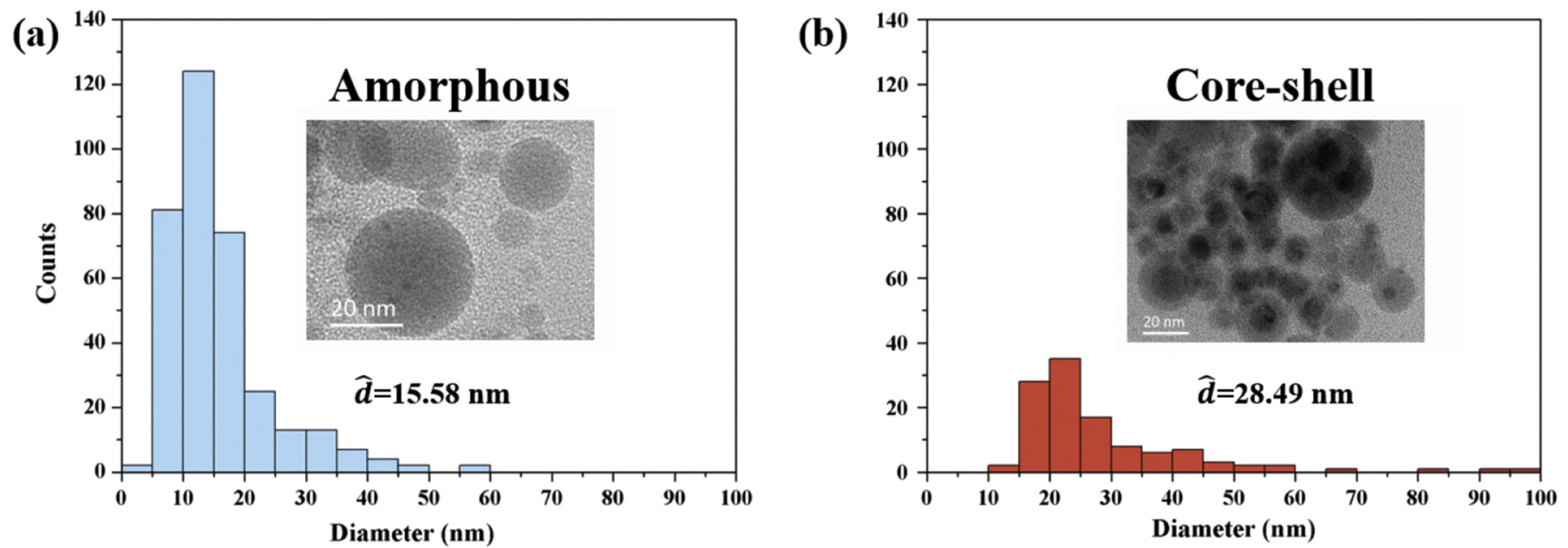

Fig. 6. Size distribution of (a) amorphous and (b) core-shell nanoparticles.
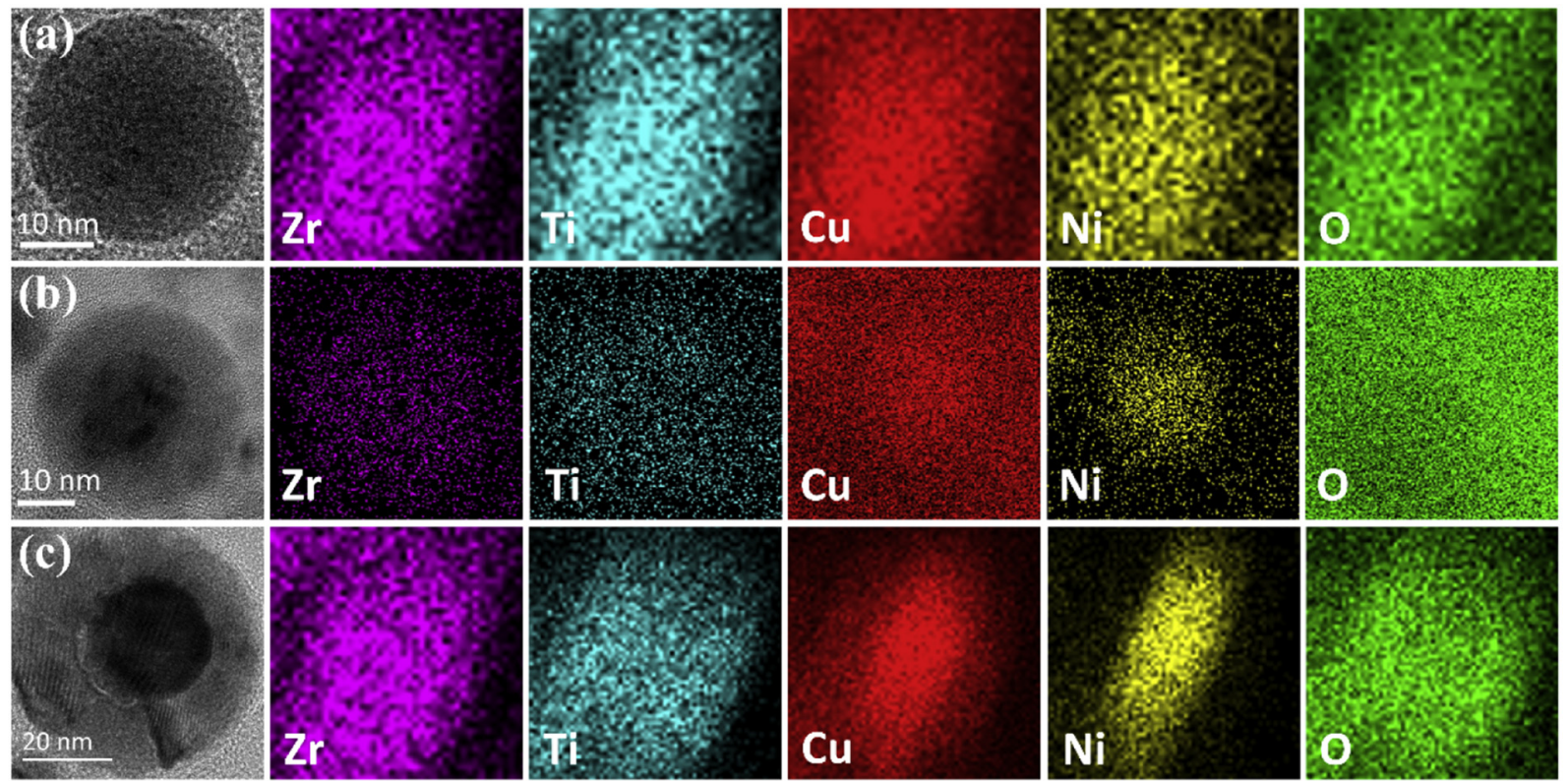

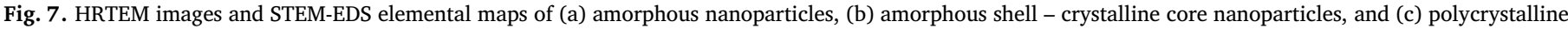
core - shell nanoparticles.

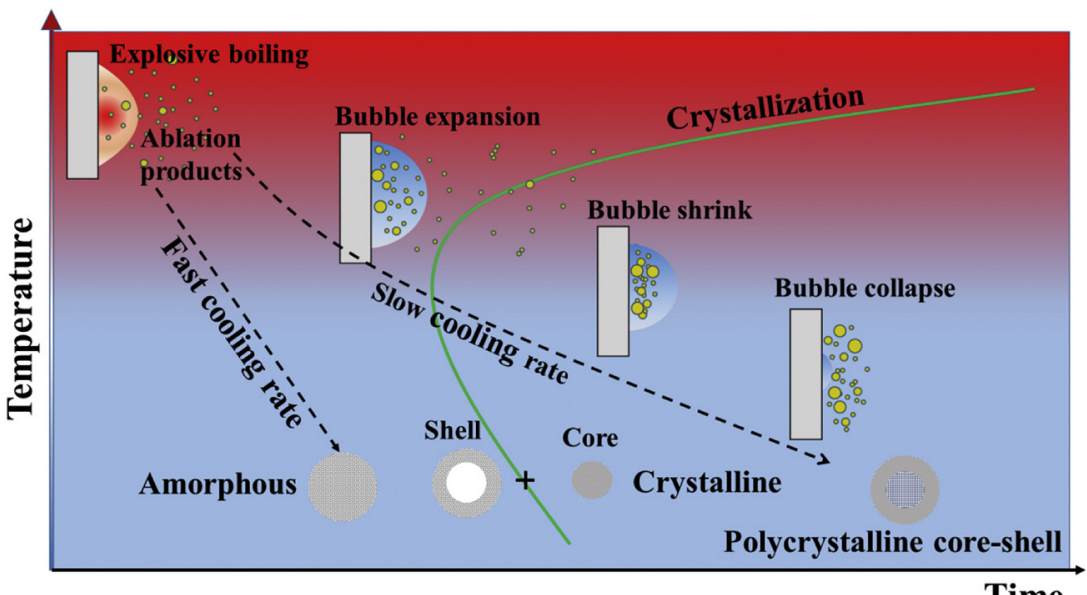

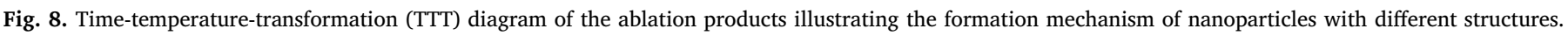


detected because of its low atomic mass. As mentioned above, the additional high-concentration of the element $\mathrm{O}$ results from the laser-assisted chemical reaction between nanoparticles and confined water or its vapor [33]. For the core-shell nanoparticles in Fig. 7b and c, there are some evidences of compositional segregation. The contents of elements $\mathrm{Cu}$ and $\mathrm{Ni}$, due to the positive mixing enthalpy [43], tends to concentrate in the crystalline core, although the absolute stoichiometry is not determined. The full polycrystalline nanoparticles show a more intensive $\mathrm{Cu}-\mathrm{Ni}$ segregation. Actually, such a decomposition or phase separation is detrimental to the GFA of metallic glass, and usually acts as a trigger of crystallization $[44,45]$. In addition, mixing of $\mathrm{O}$ will significantly reduce the GFA of the Vitreloy 1 [46].

At last, we briefly discuss the formation mechanism of the obtained nanoparticles, which is illustrated in Fig. 8 as a time-temperaturetransformation (TTT) diagram of the ablation products. In summary, the pathways by which the nanoparticles enter the water may determine their ultimate structures. It is believed that the amorphous nanoparticles belong to the ablation products that directly enter the water before the bubble formation. Based on previous studies [14,15] and our present observations (Fig. 2), this part of ablation products could experience an extremely rapid cooling at a rate higher than $10^{7} \mathrm{~K} / \mathrm{s}$. This cooling rate is much larger than the critical value $(\sim 1 \mathrm{~K} / \mathrm{s})$ for the glass formation of Vitreloy 1 [47]. Furthermore, the surrounding water decreases the possibility of the coalescence of ablation products. Both factors favor the formation of amorphous nanoparticles by avoiding the TTT nose, which compensates the detrimental effect due to the oxidation.

According to the analysis presented above, the results indicate that the core-shell nanoparticles come from the ablation products trapped by the cavitation bubble. Their multiple core-shell structures are closely related to the complex thermo-mechanical conditions inside the bubble. First, the thermal conductivity inside the bubble is lower than that in the surrounding water. The stored nanoparticles will remain hot for a longer time [48], which provides sufficient time for phase separation and subsequent crystallization. Second, both pressure and temperature inside the bubble evolve with the bubble dynamics. They will reach a peak at the latter stage of bubble shrinkage [31,49-51], usually exceeding $1 \mathrm{GPa}$ and $10^{3} \mathrm{~K}$, respectively. This HPT condition in the bubble decreases the potential barrier of nucleation of crystals [52]. On the other hand, such an extreme environment is propitious to the oxidation reaction of ablation products. All these factors mentioned above significantly reduce the GFA of nanoparticles, and meanwhile leads to a relatively low cooling rate. Furthermore, the higher concentration of ablation species in the bubble promotes the aggregation and growth of the nanoparticle. Therefore, the nanoparticles inside the bubble will experience a partial or even full crystallization and reach a larger size. The cooling rate of the outer layer of nanoparticles is usually higher than that of the inner core. This explains why most of the core-shell nanoparticles have an amorphous shell, but a crystalline core. If the cooling rate is low enough, it is possible to form the completely polycrystalline core-shell nanoparticles, as observed in Fig. $5 \mathrm{~g}$ and h. The single-crystal-core nanoparticles may well come from the ablation products that are transitorily trapped by the bubble and then quickly penetrate it.

\section{Conclusions}

Single PLA of the Vitreloy 1 target was performed in water by using a nanosecond laser. Explosive boiling of the target and cavitation bubble formation of the water were observed, which indicates the presence of different pathways by which the ablation products enter the water. An intriguing finding is that a rich variety of nanoparticles are generated from these ablation products, which provides further evidence for the explosive boiling. We demonstrate that the obtained nanoparticles are alternatively full amorphous or partially or even totally crystalline. The amorphous nanoparticles are compositionally and structurally uniform, while the latter nanoparticles display interesting core-shell structures with a compositional segregation. We suggest that the diversity of nanoparticles strongly depends on the spatiotemporal relation of explosive boiling with the dynamics of cavitation bubble. The amorphous nanoparticles can be formed if the ablation products directly enter the water before the bubble forms. The core-shell nanoparticles are usually trapped by the bubble where a complex thermomechanical environment provides a possibility of partially or full crystallization.

Although the purpose of the present study itself is not to produce nanoparticles, we can learn some useful suggestions for producing nanoparticles in the future work. Based on our findings, during the underwater PLA, the cavitation bubble seems adverse to the formation of amorphous nanoparticles, even if a metallic glass with good GFA is used as the target. This explains why amorphous nanoparticles have only rarely been obtained in previous underwater-PLA studies. In order to obtain amorphous nanoparticles, an effective strategy might be based on the delay or even inhibition of cavitation nucleation as far as possible, thus permitting the vast majority of ablation products to directly enter the liquid. Therefore, suitable liquids with lower nucleation rates should be carefully chosen as the confined media. At the same time, the chosen liquid should have a relatively high thermal conductivity and a high chemical stability. On the contrary, partially or full crystallized nanoparticles with unique core-shell structures are preferably available. In addition, the detailed effects of laser parameters and target surface on the formation of nanoparticles deserve to be further studied in our latter work.

Supplementary data to this article can be found online at https:// doi.org/10.1016/j.jnoncrysol.2019.05.009.

\section{Acknowledgments}

This work was supported by the National Natural Science Foundation of China (Grant Nos. 11522221 and 11790292).

\section{References}

[1] T.H. Maiman, Stimulated optical radiation in ruby, Nature 187 (1960) 493-494.

[2] W. Klement, R.H. Willens, P. Duwez, Non-crystalline structure in solidified goldsilicon alloys, Nature 187 (1960) 869-870.

[3] D.T.A. Matthews, V. Ocelik, J.T.M. de Hosson, Scratch test induced shear banding in high power laser remelted metallic glass layers, J. Mater. Res. 22 (2007) 460-470.

[4] D.T.A. Matthews, V. Ocelík, J.T.M. de Hosson, Tribological and mechanical properties of high power laser surface-treated metallic glasses, Mater. Sci. Eng. A 471 (2007) 155-164.

[5] H. Sun, K.M. Flores, Microstructural analysis of a laser-processed Zr-based bulk metallic glass, Metall. Mater. Trans. A 41 (2010) 1752-1757.

[6] H.Q. Sun, K.M. Flores, Spherulitic crystallization mechanism of a Zr-based bulk metallic glass during laser processing, Intermetallics 43 (2013) 53-59.

[7] J. Fu, Y. Zhu, C. Zheng, R. Liu, Z. Ji, Effect of laser shock peening on mechanical properties of Zr-based bulk metallic glass, Appl. Surf. Sci. 313 (2014) 692-697.

[8] Y. Cao, X. Xie, J. Antonaglia, B. Winiarski, G. Wang, Y.C. Shin, P.J. Withers, K.A. Dahmen, P.K. Liaw, Laser shock peening on Zr-based bulk metallic glass and its effect on plasticity: experiment and modeling, Sci. Rep. 5 (2015) 10789.

[9] Y. Zhu, J. Fu, C. Zheng, Z. Ji, Effect of nanosecond pulse laser ablation on the surface morphology of Zr-based metallic glass, Opt. Laser Technol. 83 (2016) $21-27$.

[10] H. Huang, M.Q. Jiang, J.W. Yan, Softening of Zr-based metallic glass induced by nanosecond pulsed laser irradiation, J. Alloys Compd. 754 (2018) 215-221.

[11] H. Huang, M.Q. Jiang, J.W. Yan, The coupling effects of laser thermal shock and surface nitridation on mechanical properties of Zr-based metallic glass, J. Alloys Compd. 770 (2019) 864-874.

[12] Y. Liu, M.Q. Jiang, G.W. Yang, Y.J. Guan, L.H. Dai, Surface rippling on bulk metallic glass under nanosecond pulse laser ablation, Appl. Phys. Lett. 99 (2011) 191902.

[13] Y. Liu, M.Q. Jiang, G.W. Yang, J.H. Chen, Y.J. Guan, L.H. Dai, Saffman-Taylor fingering in nanosecond pulse laser ablating bulk metallic glass in water, Intermetallics 31 (2012) 325-329.

[14] M.Q. Jiang, Y.P. Wei, G. Wilde, L.H. Dai, Explosive boiling of a metallic glass superheated by nanosecond pulse laser ablation, Appl. Phys. Lett. 106 (2015) 021904

[15] E. Williams, E.B. Brousseau, Nanosecond laser processing of Zr 41.2 Ti 13.8 Cu 12.5 Ni 10 Be 22.5 with single pulses, J. Mater. Process. Technol. 232 (2016) 34-42.

[16] E. Williams, N. Lavery, Laser processing of bulk metallic glass: a review, J. Mater. Process. Technol. 247 (2017) 73-91.

[17] X. Zeng, X.L. Mao, R. Greif, R.E. Russo, Experimental investigation of ablation 
efficiency and plasma expansion during femtosecond and nanosecond laser ablation of silicon, Appl. Phys. A Mater. Sci. Process. 80 (2005) 237-241.

[18] J. Wu, W. Wei, X. Li, S. Jia, A. Qiu, Infrared nanosecond laser-metal ablation in atmosphere: initial plasma during laser pulse and further expansion, Appl. Phys. Lett. 102 (2013) 164104.

[19] F. Ma, J. Yang, X. Zhu, C. Liang, H. Wang, Femtosecond laser-induced concentric ring microstructures on Zr-based metallic glass, Appl. Surf. Sci. 256 (2010) 3653-3660.

[20] H.P. Guo, Q.H. Lou, S.C. Chen, N.H. Cheung, Z.Y. Wang, P.K. Lin, Beam-deflection study of shock wave formation and propagation in gas ambient during uv laser ablation of solid material, Opt. Commun. 98 (1993) 220-224.

[21] T.T.P. Nguyen, R. Tanabe, Y. Ito, Comparative study of the expansion dynamics of laser-driven plasma and shock wave in in-air and underwater ablation regimes, Opt. Laser Technol. 100 (2018) 21-26.

[22] M.Q. Jiang, X.Q. Wu, Y.P. Wei, G. Wilde, L.H. Dai, Cavitation bubble dynamics during pulsed laser ablation of a metallic glass in water, Extreme Mech. Lett. 11 (2017) 24-29.

[23] H. Zeng, X.W. Du, S.C. Singh, S.A. Kulinich, S. Yang, J. He, W. Cai, Nanomaterials via laser ablation/irradiation in liquid: a review, Adv. Funct. Mater. 22 (2012) 1333-1353.

[24] N. Patra, K. Akash, S. Shiva, R. Gagrani, H.S.P. Rao, V.R. Anirudh, I.A. Palani, V. Singh, Parametric investigations on the influence of nano-second Nd 3+: YAG laser wavelength and fluence in synthesizing NiTi nano-particles using liquid assisted laser ablation technique, Appl. Surf. Sci. 366 (2016) 104-111.

[25] D. Zhang, B. Gökce, S. Barcikowski, Laser synthesis and processing of colloids: fundamentals and applications, Chem. Rev. 117 (2017) 3990-4103.

[26] J. Xiao, P. Liu, C.X. Wang, G.W. Yang, External field-assisted laser ablation in liquid: an efficient strategy for nanocrystal synthesis and nanostructure assembly, Prog. Mater. Sci. 87 (2017) 140-220.

[27] A. Peker, W.L. Johnson, A highly processable metallic glass: Zr41.2Ti13.8Cu12.5Ni10.0Be22.5, Appl. Phys. Lett. 63 (1993) 2342-2344.

[28] T. Tsuji, D.H. Thang, Y. Okazaki, M. Nakanishi, Y. Tsuboi, M. Tsuji, Preparation of silver nanoparticles by laser ablation in polyvinylpyrrolidone solutions, Appl. Surf. Sci. 254 (2008) 5224-5230.

[29] S. Reich, P. Schönfeld, P. Wagener, A. Letzel, S. Ibrahimkutty, B. Gökce, S. Barcikowski, A. Menzel, T. dos Santos Rolo, A. Plech, Pulsed laser ablation in liquids: impact of the bubble dynamics on particle formation, J. Colloid Interface Sci. 489 (2017) 106-113.

[30] W. Soliman, T. Nakano, N. Takada, K. Sasaki, Modification of Rayleigh-Plesset theory for reproducing dynamics of cavitationbubbles in liquid-phase laser ablation, Jpn. J. Appl. Phys. 49 (2010) 116202.

[31] J. Lam, J. Lombard, C. Dujardin, G. Ledoux, S. Merabia, D. Amans, Dynamical study of bubble expansion following laser ablation in liquids, Appl. Phys. Lett. 108 (2016) 074104.

[32] X. Xu, Phase explosion and its time lag in nanosecond laser ablation, Appl. Surf. Sci. 197 (2002) 61-66.

[33] B.E.B. Al-Jumaili, Z.A. Talib, A. Zakaria, A. Ramizy, N.M. Ahmed, S.B. Paiman, J.L. Ying, I.B. Muhd, H. Baqiah, Impact of ablation time on $\mathrm{Cu}$ oxide nanoparticle green synthesis via pulsed laser ablation in liquid media, Appl. Phys. A Mater. Sci. Process. 124 (2018) 577.

[34] M.Q. Jiang, G.H. Duan, L.H. Dai, Metallic glass nanofilms, J. Non-Cryst. Solids 357
(2011) 1621-1627.

[35] K.S. Nakayama, Y. Yokoyama, G. Xie, Q. Zhang, M. Chen, T. Sakurai, A. Inoue, Metallic glass nanowire, Nano Lett. 8 (2008) 516-519.

[36] G. Kumar, H.X. Tang, J. Schroers, Nanomoulding with amorphous metals, Nature 457 (2009) 868-872.

[37] Y. Liu, K. Wang, D. Pan, H. Wang, K. Nakayama, A. Inoue, W. Wang, M. Chen, Plastic deformation-assisted synthesis of metallic glass nanostructures, Mater. Trans. 50 (2009) 1890-1893.

[38] X.X. Xia, W.H. Wang, A.L. Greer, Plastic zone at crack tip: a nanolab for formation and study of metallic glassy nanostructures, J. Mater. Res. 24 (2009) 2986-2992.

[39] K.S. Nakayama, Y. Yokoyama, T. Ono, M.W. Chen, K. Akiyama, T. Sakurai, A. Inoue Controlled formation and mechanical characterization of metallic glassy nanowires, Adv. Mater. 22 (2010) 872-875.

[40] N. Chen, D. Wang, P.F. Guan, H.Y. Bai, W.H. Wang, Z.J. Zhang, H. Hahn, H. Gleiter Direct observation of fast surface dynamics in sub-10-nm nanoglass particles, Appl. Phys. Lett. 114 (2019) 043103.

[41] J. Jing, A. Kramer, R. Birringer, H. Gleiter, U. Gonser, Modified atomic structure in a Pd-Fe-Si nanoglass: a Mössbauer study, J. Non-Cryst. Solids 113 (1989) 167-170.

[42] H. Gleiter, Our thoughts are ours, their ends none of our own: are there ways to synthesize materials beyond the limitations of today? Acta Mater. 56 (2008) 5875-5893.

[43] I. Martin, T. Ohkubo, M. Ohnuma, B. Deconihout, K. Hono, Nanocrystallization of Zr41.2Ti13.8Cu12.5Ni10.0Be22.5 metallic glass, Acta Mater. 52 (2004) 4427-4435.

[44] X.L. Wang, J. Almer, C.T. Liu, Y.D. Wang, J.K. Zhao, A.D. Stoica, D.R. Haeffner, W.H. Wang, In situ synchrotron study of phase transformation behaviors in bulk metallic glass by simultaneous diffraction and small angle scattering, Phys. Rev. Lett. 91 (2003) 265501.

[45] X.J. Liu, X.D. Hui, G.L. Chen, M.H. Sun, In situ synchrotron SAXS study of nanocrystallization in Zr65Ni25Ti10 metallic glass, Intermetallics 16 (2008) 10-15.

[46] C.H. Wong, C.H. Shek, Difference in crystallization kinetics of Zr41Ti14Cu12.5Ni10Be22.5 bulk metallic glass under different oxidizing environments, Intermetallics 12 (2004) 1257-1259.

[47] J. Schroers, A. Masuhr, W.L. Johnson, R. Busch, Pronounced asymmetry in the crystallization behavior during constant heating and cooling of a bulk metallic glass-forming liquid, Phys. Rev. B 60 (1999) 11855-11858.

[48] T. Tsuji, Y. Tsuboi, N. Kitamura, M. Tsuji, Microsecond-resolved imaging of lase ablation at solid-liquid interface: investigation of formation process of nano-size metal colloids, Appl. Surf. Sci. 229 (2004) 365-371.

[49] I. Akhatov, O. Lindau, A. Topolnikov, R. Mettin, N. Vakhitova, W. Lauterborn, Collapse and rebound of a laser-induced cavitation bubble, Phys. Fluids 13 (2001) 2805-2819.

[50] A. De Giacomo, M. Dell'Aglio, A. Santagata, R. Gaudiuso, O. De Pascale, P. Wagener, G.C. Messina, G. Compagnini, S. Barcikowski, Cavitation dynamics of laser ablation of bulk and wire-shaped metals in water during nanoparticles production, Phys. Chem. Chem. Phys. 15 (2013) 3083-3092.

[51] M. Dell'Aglio, R. Gaudiuso, O. De Pascale, A. De Giacomo, Mechanisms and processes of pulsed laser ablation in liquids during nanoparticle production, Appl. Surf. Sci. 348 (2015) 4-9.

[52] B. Yao, S.E. Liu, L. Liu, L. Si, W. Su, Y. Li, Mechanism of mechanical crystallization of amorphous Fe-Mo-Si-B alloy, J. Appl. Phys. 90 (2001) 1650-1654. 